\title{
AVALIAÇÃO EPIDEMIOLÓGICA DA HANSENÍASE E DOS SERVIÇOS RESPONSÁVEIS POR SEU ATENDIMENTO EM RIBEIRÃO PRETO - SP NO ANO DE 1992
}

\author{
EPIDEMIOLOGICAL EVALUATION OF LEPROSY AND LEPROSY HEALTH \\ SERVICES IN RIBEIRÃO PRETO CITY, BRAZIL, IN 1992
}

\begin{abstract}
Sátiro Nakamura Oliveira ${ }^{1}$; Gisele Vieira Hennemann ${ }^{1}$; Flávio Luís Fantini Ferreira ${ }^{2}$; Sílvia Amorim Azevedo'; Aldaísa Cassanho Forster ${ }^{3}$
\end{abstract}

Médico Residente ${ }^{1}$ do Hospital das Clínicas de Ribeirão Preto. Pós-Graduando²; Docente ${ }^{3}$ junto ao Departamento de Medicina Social da Faculdade de Medicina de Ribeirão Preto da Universidade de São Paulo.

CoRRESPONDÊNCIA: Departamento de Medicina Social da Faculdade de Medicina de Ribeirão Preto - Campus Universitário - CEP:14048-900 Ribeirão Preto - SP. Fax (016) 633-5839

OLIVEIRA SN et al. Avaliação epidemiológica da hanseníase e dos serviços responsáveis por seu atendimento em Ribeirão Preto - SP no ano de 1992. Medicina, Ribeirão Preto, 29: 114-122, jan/mar 1996.

RESUMO: A partir de dados registrados na planilha epidemiológica de hanseníase, instituída pelo Ministério da Saúde, sobre os casos atendidos no Município de Ribeirão Preto-S.P., buscou-se traçar um perfil epidemiológico da hanseníase, no ano de 1992, e avaliar a eficácia dos serviços responsáveis pela assistência aos hansenianos.

Encontrou-se um coeficiente de prevalência de 1,72/1000 habitantes, pouco abaixo do coeficiente nacional de 1992 (1,78/1000 habitantes), e superior àquele preconizado pela O.M.S. para definir áreas de baixa prevalência (0,2/1000 habitantes). A maioria dos casos $(65,4 \%)$ era de formas multibacilares, e o esquema terapêutico predominante foi o DNDS (apesar das recomendações do Ministério da Saúde). Detectou-se, também, que cerca da metade dos pacientes não recebeu avaliação de incapacidades durante o ano, havendo $60 \%$ dos pacientes com incapacidades de graus II e III, entre os que saíram do programa por alta, por cura e foram avaliados quanto a incapacidades, apesar do comparecimento dos pacientes ter sido satisfatório.

Com base em tais resultados inquietantes, os autores propõem a descentralização do atendimento para as Unidades Básicas de Saúde, aproximando o serviço à comunidade. Encaminhamentos a serviços de nível secundário e terciário seriam feitos apenas para determinação diagnóstica mais apurada, e na ocorrência de complicações. Grupos terapêuticos com orientação multiprofissional e multidisciplinar deveriam ser formados para discutir o estigma e formas de reintegrar, socialmente, os pacientes.

UNITERMOS: Hanseníase. Saúde Pública. Programas Nacionais de Saúde - Avaliação.

\section{INTRODUÇÃO}

A hanseníase é uma doença crônica, transmissível, de acometimento do sistema nervoso periférico e de diversos órgãos (pele, rins, fígado, sistema osteo-articular), afetados primária ou secundariamente. É causada pelo Micobacterium leprae (ou bacilo de Hansen) e tem evolução insidiosa na maior parte dos casos. Este microrganismo tem como características grande resistência ao meio ambiente: em clima quente, à temperatura de $30^{\circ} \mathrm{C}$, e úmido, é viável por quarenta dias; em condições desfavoráveis, é viável por 7 a 10 dias, com pequena patogenicidade o tempo de multiplicação é de 21 dias $^{1}$, em alta infectividade e virulência causa 
grande incapacidade física e discriminação social. Seu poder imunogênico é praticamente nulo, sendo a reexposição constante uma das causas de recidivas. Sua transmissão ainda não foi bem definida, mas encontra maior probabilidade de contágio entre pessoas que estejam em contato íntimo e prolongado com doentes ${ }^{2}$.

Apresenta-se numa enorme variedade de formas clínicas, determinadas pela resposta imunológica do hospedeiro ${ }^{2}$. Epidemiologicamente, a hanseníase apresenta doentes que são pólos transmissores da doença (multibacilares, aqueles com altas cargas bacilares, as formas clínicas dimorfa e virchoviana, segundo a classificação de Madri² e doentes sem capacidade de propagação da doença, com resposta imunológica mais eficiente contra o bacilo (paucibacilares, aqueles com escassa carga bacilar, formas clínicas indeterminada, tuberculóide e tuberculóide reacional ${ }^{2}$. As formas multibacilares e paucibacilares são, clinicamente, diferenciáveis pela baciloscopia da linfa ${ }^{2,3}$.

É doença de notificação compulsória ${ }^{4}$, e sua magnitude e abrangência tornam-na grave problema de saúde pública. Segundo dados da OMS em 1992, o Brasil ocupava a segunda colocação em número absoluto de casos, com coeficiente de prevalência de 1,78/1000 habitantes, sendo superado somente pela Índia. No Estado de São Paulo, cujos dados são mais confiáveis, documenta-se uma endemia de altos níveis ${ }^{1}$.

Quanto maior a prevalência das formas multibacilares na população, menos eficazes estão sendo as medidas de controle, pois a endemia está abandonada às suas tendências naturais, e o grande número de focos transmissores mantém a geração de novos doentes. A proporção entre as formas clínicas, também, é dado epidemiológico importante, uma vez que indivíduos com maior resistência à instalação da doença são aqueles em que a hanseníase manifesta-se nas formas paucibacilares. Grande proporção de doentes paucibacilares evidencia que a endemia atinge até aqueles com maior resistência à doença. A distribuição etária dos doentes é igualmente importante; sendo a hanseníase transmitida através de longos períodos de contato, é maior o número de doentes menores de 15 anos quanto maior for a exposição a focos transmissores.

$\mathrm{Na}$ assistência ao hanseniano, além do tratamento, outro cuidado assume fundamental importância, tanto na consideração da saúde do indivíduo como no aspecto de saúde pública, é a prevenção do surgimento de incapacidades. A hanseníase pode trazer sequielas, mesmo após o controle e até destruição dos bacilos de Hansen, por mecanismos imunológicos que acarretam lesões neurológicas irreversíveis. Cabe, então, ao serviço de assistência prevenir o surgimento de tais seqüelas com avaliações neurológicas periódicas, e intervindo quando necessário com o uso de corticóides, imunossupressores (a talidomida), e de fisioterapia. Avaliação feita pelo Ministério da Saúde, em 1986, mostrou incapacidades físicas em $40 \%$ do total de pacientes; entretanto, estes dados mostram apenas parte da realidade, uma vez que somente $20 \%$ das Unidades Federadas informaram estes dados, e que apenas $13 \%$ do total das mesmas contam com serviços de prevenção de incapacidades ${ }^{3}$.

O tratamento possui esquemas medicamentosos e funcionais padronizados pelo Ministério da Saúde, baseados em protocolos preconizados pela OMS. Em 1990, o antigo esquema baseado na Rifampicina e Dapsona do Departamento Nacional de Dermatologia Sanitária (DNDS) foi substituído por um esquema de drogas combinadas, a Poliquimioterapia (PQT) ${ }^{4}$, já adotada na época por vários países. Tal substituição foi justificada pelo fato de que a longa duração do antigo esquema (muitas vezes, a vida inteira) culminava em grande número de abandonos, além de estudos recentes feitos pela OMS mostrarem indícios de grande resistência do bacilo de Hansen à Dapsona. O novo esquema prevê fim do tratamento para os multibacilares em dois anos, e para os paucibacilares em seis meses, aumentando a aderência ao tratamento, e o número de altas por cura. A PQT está em expansão por todo o país; dados divulgados em 1993, mostravam a adoção da PQT em 83,9\% dos municípios e 98,4\% da população do Estado de São Paulo, sendo que o Programa Nacional de Controle da Hanseníase cobria 91,1\% dos municípios e $97,5 \%$ da população, no mesmo período. Em 1994, o Ministério da Saúde apresentou o Plano de Eliminação da Hanseníase no Brasil para o período de $1995-2000^{5}$, com base na cobertura por tratamento de $90 \%$ dos casos ativos com PQT/OMS.

\section{OBJETIVOS}

Considerando os aspectos abordados, pretendemos estudar a distribuição da hanseníase no Município de Ribeirão Preto no ano de 1992, com os seguintes objetivos:

Analisar os aspectos clínico-epidemiológicos, traçando um perfil epidemiológico da doença.

Avaliar as atividades desenvolvidas pelos serviços de saúde na assistência aos pacientes com hanseníase. 


\section{MATERIAL E MÉTODOS}

A cidade de Ribeirão Preto é uma das cidades mais prósperas do interior do Estado de São Paulo, pólo econômico, fundamentado na cultura agrícola da cana-de-açúcar e de monopólios latifundiários de laranja, café, soja e milho, com fortes setores econômicos intermediário e terciário. É sede de grande afluxo de migrantes, conforme as safras agrícolas, e apesar de ter renda "per capita" elevada (uma das maiores do país), apresenta grande desigualdade sócio-econômica que se aprofunda ano a ano. Estudos indicam que o salário, médio no setor privado, é pouco superior a dois salários-mínimos, situação que é pior na área primária, enquanto a renda "per capita" da elite econômica é extremamente elevada ${ }^{6}$. Segundo censo de 1991, apresentava 436.682 habitantes, e PIB de aproximadamente 10 milhões de dólares ${ }^{7}$.

É, ainda, centro cultural e educacional, com escolas privadas e públicas de alto nível, abrigando quatro universidades, entre elas um campus da USP. A Faculdade de Medicina de Ribeirão Preto é centro de referência tecnológico e científico, e seu Hospital das Clínicas, polariza a atenção médica de nível terciário de todo o Nordeste paulista.

Toda a demanda de hanseníase concentrava-se, em 1992, em quatro unidades: Núcleo de Gestão Ambulatorial-59, Centro de Saúde Escola Sumarezinho, UBDS Vila Virgínia e UBS Bonfim Paulista. O atendimento é regionalizado e há um médico dermatologista responsável pelo atendimento, tanto de doentes hansenianos, quanto de dermatologia geral, em cada unidade ${ }^{8}$.

O Centro de Saúde Escola, além do serviço de dermatologia, conta com as atividades extracurriculares dos acadêmicos da "Liga de Combate à Hanseníase - Luiz Marino Bechelli" da Faculdade de Medicina de Ribeirão Preto-USP.

Para cumprir o primeiro objetivo, foram coletadas as seguintes informações das planilhas epidemiológicas de hanseníase do ERSA-50: idade, sexo, procedência, forma clínica, esquema medicamentoso, avaliação de incapacidades, comparecimento, atividade da doença, baciloscopias realizadas, situação de tratamento, registros dos comunicantes, casos novos e saídas, por unidade de atendimento. Foram utilizados os indicadores epidemiológicos recomendados pelo Ministério da Saúde para o acompanhamento das atividades de controle da hanseníase ${ }^{4}$ : a) Coeficiente de registro anual de casos novos:

$$
\frac{\text { Casos novos registrados no ano }}{\text { População em primeiro de julho }} \quad \text { X } 100.000
$$

Utilidade: determinar a tendência secular e medir a intensidade das medidas de detecção.

b) Coeficiente de prevalência, registrada no final do ano:

$$
\frac{\text { Casos em registro ativo em } 31 \text { de dezembro }}{\text { População em } 31 \text { de dezembro }} \times 1000
$$

Utilidade: medir a magnitude da doença.

c) Proporção de menores de 15 anos entre casos novos registrados no ano;

$\frac{\text { Casos registrados no ano em menores de } 15 \text { anos }}{\text { Total de casos novos registrados no ano }}$ X 100

Utilidade: determinar a tendência da endemia.

d) Proporção de casos novos registrados no ano de forma $\mathrm{T}$, em relação aos casos $\mathrm{V}, \mathrm{D}$ e T:

$\frac{\text { Casos novos registrados no ano de forma T }}{\text { Casos novos registrados no ano de formas V, D e T }} \quad$ X 100

Utilidade: determinar a tendência da endemia.

e) Proporção de doentes com deformidades entre os casos novos registrados e avaliados:

$$
\begin{gathered}
\text { Casos novos registrados com grau de } \\
\text { incapacidade II e III }
\end{gathered}
$$

Utilidade: estimar a eficiência das atividades para detecção precoce de casos.

f) Proporção de doentes com deformidades, entre os casos em registro ativo avaliados:

Doentes em registro ativo, com grau de incapacidade X 100

$$
\begin{aligned}
& \text { Doentes, em registro ativo, com } \\
& \text { grau de incapacidade avaliado }
\end{aligned}
$$

Utilidade: estimar a transcendência do problema no ano de referência.

g) Proporção de casos V e D com baciloscopia positiva:

$\underline{\text { Casos V e D cuja última baciloscopia do ano foi positiva }}$ X 100 Casos V e D submetidos a baciloscopia no ano

Utilidade: estimar a eficácia do tratamento quimioterápico nos casos contagiantes, e a prevalência das fontes de contágio. 
h) Proporção de pacientes curados com seqüelas:

$$
\frac{\text { Altas por cura, no ano, com grau de incapacidade II e III }}{\text { Altas por cura, no ano, com grau de incapacidade }} 100
$$

Utilidade: estimar a capacidade que os serviços de saúde têm de curar o paciente com o mínimo de seqüelas possíveis.

Para cumprir o segundo objetivo, foram utilizados o conceito de eficácia ${ }^{9}$ e os indicadores operacionais, recomendados pelo Ministério da Saúde para o acompanhamento das atividades de controle da hanseníase ${ }^{4}$ :

a) Proporção de casos novos registrados com grau de capacidade avaliado:

$\begin{gathered}\text { Doentes novos registrados com } \\ \text { grau de incapacidade avaliado }\end{gathered}$
Total de casos novos, registrados no ano

Utilidade: medir a qualidade do atendimento dos serviços.

b) Proporção de doentes, em registro ativo, com grau de incapacidade avaliado no ano de referência:

\begin{tabular}{l}
$\begin{array}{l}\text { Doentes em registro ativo, em 31/12, } \\
\text { com grau de incapacidade avaliado }\end{array} \times 100$ \\
\hline
\end{tabular}

Total de doentes em registro ativo em 31.12 ,

Utilidade: medir a qualidade do atendimento.

c) Proporção de doentes $\mathrm{V}$ e $\mathrm{D}$, em registro ativo, submetidos à baciloscopia no ano:

Doentes V e D submetidos à baciloscopia no ano X 100

Doentes $\mathrm{V}$ e D em registro ativo, 31/12

Utilidade: medir a qualidade do atendimento.

d) Proporção de doentes em registro ativo, em 31/12, com comparecimento regular:

Doentes em registro ativo, em 31/12, com comparecimento regular

Doentes em registro ativo em 31/12

Utilidade: medir a regularidade com que os pacientes comparecem às consultas aprazadas. Medir a efetividade das ações preconizadas.

e) Proporção de pacientes curados, com grau de capacidade avaliado:

\footnotetext{
Pacientes com alta por cura, no ano, com grau de incapacidade avaliado
}

Total de pacientes com alta por cura, no ano X 100

Utilidade: medir a qualidade do atendimento. f) Proporção de examinados, entre os contatos intradomiciliares de casos novos:

Contatos intradomiciliares de casos novos, no ano

Total de contatos intradomiciliares de casos novos X 100

Utilidade: medir eficiência das medidas de vigilância.

\section{RESULTADOS E DISCUSSÃO}

É bom lembrar que esta avaliação ocorre após dois anos da implantação do Sistema Único de Saúde (SUS) no país.

Em Ribeirão Preto, houve a municipalização da Vigilância Epidemiológica, tendo o nível municipal assumido a maior parte das atividades para as doenças sob vigilância ${ }^{10}$.

No tocante à hanseníase, o processo deu-se de forma incompleta, pois os serviços responsáveis pelo Programa de Controle permaneciam em 1992 em Unidades de Saúde Estaduais (CSE e NGA-50) e outras duas municipais (UBDS Bonfim Paulista, antes estadual, e UBDS Vila Virgínia).

É inegável, porém, que à medida que o SUS avança, a acessibilidade aos serviços de saúde e os contatos com as equipes de saúde tendem a aumentar as "chances" de diagnóstico e tratamento precoce para os hansenianos ${ }^{11}$.

Ocorre, na realidade, falta de integração entre as equipes das UBSs e das Unidades de nível secundário, onde estão centradas as atividades programáticas da Hanseníase. Assim, o paciente é seguido na UBS para problemas de saúde quaisquer, dirigindo-se para o seguimento da doença de base para a referência secundária. A equipe do programa passa a ser responsável por atividades de saúde típicas de atenção básica e outras da esfera secundária, com repercussão na eficácia das ações.

No ano de 1992, para um total de 823 pacientes residentes no município de Ribeirão Preto, houve registro ativo de 754 pacientes até o final do ano, dos quais 95 eram casos novos, resultando em coeficientes de prevalência de 1,72 hansenianos (por 1000 habitantes) e de registro de casos novos de 21,75/100.000 habitantes. Com esses resultados, Ribeirão Preto classifica-se como região endêmica, sem controle para a hanseníase, com coeficiente de prevalência maior que aquele recomendado pela O.M.S para definir áreas de alta prevalência e apenas pouco abaixo ao observado no país (1,78/1000 habitantes), além de uma alta taxa de detecção de casos novos. Altas taxas de detecção 
podem evidenciar serviços de saúde eficientes, mas os valores encontrados permitem a consideração da hipótese de que a endemia, fora de controle, gera grande número de doentes. Nos últimos anos, os serviços de atendimento a hansenianos no município melhoraram sua organização, o que pode ter contribuído em parte para o aumento da detecção. A proporção de doentes com deformidades, entre os casos novos registrados e avaliados $(39,28 \%)$, foi alta (padrão do Ministério da Saúde), indicando falta de controle da endemia, pois o diagnóstico é feito tardiamente. Rojas et al (1994) observaram diferença no período de tempo que decorreu desde o início do quadro da doença e o diagnóstico de certeza entre pacientes de duas cidades cubanas com acesso igual aos serviços de saúde ${ }^{11}$.

Medida importante na detecção de casos novos da hanseníase; a busca ativa do doente entre os comunicantes intradomiciliares dos casos atingiu apenas $60 \%$ dos casos novos. É falha importante, no tocante ao controle da endemia.

Dos 823 pacientes registrados, 60,6\% (499) eram do sexo masculino e $39,4 \%$ eram do sexo feminino (Tabela I).

A maioria dos doentes é adulta, sendo que do total de casos, 59,0\% têm entre 40 e 69 anos, e 20,6\%, entre 60 e 69 anos de idade (Tabela I). Cerca de 3,1\% dos casos novos tinham menos de quinze anos de idade, abaixo do parâmetro do Ministério da Saúde para essa faixa etária, indicando que a endemia poupa as faixas etárias menores.
$\mathrm{Na}$ Tabela II, temos os coeficientes de prevalência por faixa etária, em relação à população do município de Ribeirão Preto; notando-se que foram excluídos no cálculo os pacientes que saíram de registro durante o ano de 1992, por cura, óbito, transferências ou outros motivos. Podemos perceber que a prevalência da hanseníase nessa população aumenta nas faixas etárias dos adultos e idosos, com pequena queda a partir dos setenta anos, situando seu pico na faixa de 60 a 69 anos. Parece mostrar que a doença manifesta-se realmente, após vários anos de exposição, havendo "claro" outros fatores envolvidos nessa questão.

Encontrou-se que cerca de $65,4 \%$ dos pacientes eram formas multibacilares, e $34,6 \%$, paucibacilares (Tabela I). Um bom indicador epidemiológico para determinar a tendência da endemia é analisar a proporção entre paucibacilares e multibacilares, através da proporção de casos novos de forma $\mathrm{T}$, registrados no ano em relação ao total dos casos nas formas clínicas V, D e T, que foi igual a 39,28\%, um valor considerado no limite superior da faixa média. Tais dados confirmam a hipótese de que a endemia esteve fora de controle, no ano em estudo.

Uma vez diagnosticada a hanseníase, cabe ao serviço de saúde evitar que os doentes multibacilares continuem transmitindo a doença; instituir, precocemente, a terapêutica para os doentes paucibacilares, buscando a destruição dos bacilos existentes no organismo, evitando assim a evolução da doença e a instalação de seqüelas incapacitantes. A eficácia das ações

Tabela I: Distribuição dos pacientes hansenianos, atendidos no Município de Ribeirão Preto segundo forma clínica, faixa etária e sexo no ano de 1992

\begin{tabular}{|c|c|c|c|c|c|c|c|c|c|c|c|c|c|c|c|c|c|c|c|c|}
\hline \multirow{3}{*}{ Forma } & \multicolumn{20}{|c|}{ Faixas Etárias (anos) } \\
\hline & \multicolumn{2}{|c|}{$<15$} & \multicolumn{2}{|c|}{$15-19$} & \multicolumn{2}{|c|}{$20-29$} & \multicolumn{2}{|c|}{$30-39$} & \multicolumn{2}{|c|}{$40-49$} & \multicolumn{2}{|c|}{$50-59$} & \multicolumn{2}{|c|}{$60-69$} & \multicolumn{2}{|c|}{$70-79$} & \multicolumn{2}{|c|}{$80-89$} & \multicolumn{2}{|c|}{ Total } \\
\hline & $\mathrm{M}$ & $\mathrm{F}$ & $\mathrm{M}$ & $\mathrm{F}$ & $\mathrm{M}$ & $\mathrm{F}$ & $\mathrm{M}$ & $\mathrm{F}$ & $\mathrm{M}$ & $\mathrm{F}$ & $\mathrm{M}$ & $\mathrm{F}$ & $\mathrm{M}$ & $\mathrm{F}$ & $\mathrm{M}$ & $\mathrm{F}$ & $\mathrm{M}$ & $\mathrm{F}$ & $\mathrm{M}$ & $\mathrm{F}$ \\
\hline$I$ & 1 & - & 4 & 4 & 7 & 8 & 14 & 6 & 6 & 7 & 10 & 12 & 10 & 7 & 1 & 4 & 1 & 2 & 54 & 50 \\
\hline$T$ & 3 & 4 & 2 & 3 & 11 & 6 & 17 & 18 & 21 & 14 & 17 & 20 & 11 & 12 & 5 & 3 & 1 & 1 & 88 & 81 \\
\hline$T r$ & - & - & - & - & - & 1 & 1 & - & 3 & - & 1 & 2 & 1 & 1 & 1 & 1 & - & - & 7 & 5 \\
\hline$D$ & - & - & - & - & 4 & 3 & 5 & 6 & 5 & 5 & 16 & 9 & 7 & 6 & 6 & 6 & - & 1 & 43 & 36 \\
\hline \multirow[t]{3}{*}{$V$} & 1 & - & 4 & 2 & 25 & 12 & 42 & 19 & 46 & 24 & 63 & 35 & 76 & 39 & 37 & 16 & 13 & 5 & 307 & 152 \\
\hline & 5 & 4 & 10 & 9 & 47 & 30 & 79 & 49 & 81 & 50 & 107 & 78 & 105 & 65 & 50 & 30 & 15 & 9 & 499 & 324 \\
\hline & \multicolumn{2}{|c|}{9} & \multicolumn{2}{|c|}{19} & \multicolumn{2}{|c|}{77} & \multicolumn{2}{|c|}{128} & \multicolumn{2}{|c|}{131} & \multicolumn{2}{|c|}{185} & \multicolumn{2}{|c|}{170} & \multicolumn{2}{|c|}{80} & \multicolumn{2}{|c|}{24} & \multicolumn{2}{|c|}{823} \\
\hline
\end{tabular}


programadas para seguimento e tratamento dos pacientes pode ser avaliada, através da taxa dos doentes com baciloscopia positiva. De todos os pacientes em registro em 1992, apenas 9,7\% tinham baciloscopia positiva (Tabela III). A proporção de casos $\mathrm{V}$ e $\mathrm{D}$, com baciloscopia positiva, é um indicador mais confiável para avaliar a eficácia do tratamento e a manutenção das fontes de contágio, tendo atingido $18,86 \%$ (valor baixo, segundo os parâmetros do Ministério da Saúde). Foram submetidos à baciloscopia, no ano $86,72 \%$ dos casos V e D em registro ativo (ou seja, 13,28\% "dos doentes multibacilares registrados" não foram submetidos à baciloscopia). Pode-se afirmar que, apesar da endemia hansênica estar em altos níveis na população entre os doentes registrados, os serviços de controle efetuam ações eficazes na negativação das baciloscopias. Tal eficácia pode ser, em parte, atribuída à instituição do esquema de PQT.

Tabela II - Coeficientes de Prevalência de Hanseníase por faixa etária no Município de Ribeirão Preto no ano de 1992.

\begin{tabular}{lrrc}
\hline $\begin{array}{l}\text { Faixa } \\
\text { Etária }\end{array}$ & Pacientes & População & $\begin{array}{c}\text { Coeficientes de } \\
\text { Prevalência } \\
\text { (por } 1000 \text { hab.) }\end{array}$ \\
\hline$<15$ & 7 & 130.410 & 0,05 \\
15 a 19 & 18 & 38.171 & 0,47 \\
20 a 29 & 71 & 77.990 & 0,91 \\
30 a 39 & 119 & 71.196 & 1,67 \\
40 a 49 & 122 & 48.102 & 2,53 \\
50 a 59 & 175 & 33.218 & 5,26 \\
60 a 69 & 156 & 22.749 & 6,86 \\
70 a 79 & 68 & 10.857 & 6,26 \\
80 a 89 & 18 & 3.989 & 4,51 \\
\hline
\end{tabular}

\begin{tabular}{|c|c|c|c|}
\hline Baciloscopia & Masculino & Feminino & Total \\
\hline POSITIVA & $\begin{array}{c}58 \\
(11,6)\end{array}$ & $\begin{array}{c}22 \\
(6,8)\end{array}$ & $\begin{array}{c}80 \\
(9,7)\end{array}$ \\
\hline NEGATIVA & $\begin{array}{c}313 \\
(62,7)\end{array}$ & $\begin{array}{c}209 \\
(64,5)\end{array}$ & $\begin{array}{c}522 \\
(63,4)\end{array}$ \\
\hline Sem baciloscopia & $\begin{array}{c}111 \\
(22,2)\end{array}$ & $\begin{array}{c}76 \\
(23,4)\end{array}$ & $\begin{array}{c}187 \\
(22,7)\end{array}$ \\
\hline Sem informação & $\begin{array}{c}17 \\
(3,4)\end{array}$ & $\begin{array}{c}17 \\
(5,2)\end{array}$ & $\begin{array}{c}34 \\
(4,1)\end{array}$ \\
\hline Total & 499 & 324 & 823 \\
\hline
\end{tabular}

Com relação à terapêutica medicamentosa dos hansenianos nos serviços de Ribeirão Preto (Tabela IV), o levantamento mostrou que os serviços não adotaram a PQT como regime terapêutico predominante, contrário à atual tendência nacional e mundial. Apenas $12,6 \%$ dos doentes estavam sob tal esquema, enquanto que a maioria $(80,5 \%)$ ainda estava sob o antigo esquema DNDS. É ,ainda, digno de nota a proporção de pacientes sem tratamento ou sem informações registradas $(6,2 \%)$. Tais fatos podem ser atribuídos à resistência por parte dos médicos e outros profissionais de saúde à adoção do novo esquema proposto. A PQT trouxe a necessidade de reformular a assistência aos hansenianos: os retornos trimestrais passaram a ser mensais, com períodos de permanência na Unidade por duas horas, com necessárias reformulações do espaço físico, recursos humanos e da própria assistência. O acompanhamento intensivo dos pacientes acarreta aos serviços cobrir outras necessidades dos doentes, inclusive causadas por dificuldades sócio-econômicas (a assiduidade às doses mensais depende de dispensa do trabalho e de gastos com transporte). Há que se considerar, também, o estigma da doença, que torna difícil o controle da hanseníase; a mudança da terapêutica permitiu abreviar o tratamento prolongado para um período de dois anos (decreto do Ministério da Saúde), para os multibacilares, e de seis meses para os paucibacilares. Receios em relação ao uso das medicações associadas igualmente, foram responsáveis pela resistência à adoção do novo esquema terapêutico.

\begin{tabular}{|c|c|c|c|}
\hline Terapêutica & masculino & feminino & Total \\
\hline PQT & $\begin{array}{c}67 \\
(13,4)\end{array}$ & $\begin{array}{c}37 \\
(11,4)\end{array}$ & $\begin{array}{c}104 \\
(12,6)\end{array}$ \\
\hline DNDS & $\begin{array}{c}405 \\
(81,1)\end{array}$ & $\begin{array}{c}258 \\
(79,6)\end{array}$ & $\begin{array}{c}663 \\
(80,5)\end{array}$ \\
\hline Outros & 0 & $\begin{array}{c}5 \\
(1,5)\end{array}$ & $\begin{array}{c}5 \\
(0,6)\end{array}$ \\
\hline sem terapêutica & $\begin{array}{c}9 \\
(1,8)\end{array}$ & $\begin{array}{c}13 \\
(4,0)\end{array}$ & $\begin{array}{c}22 \\
(2,7)\end{array}$ \\
\hline sem informação & $\begin{array}{c}18 \\
(3,6)\end{array}$ & $\begin{array}{c}11 \\
(3,4)\end{array}$ & $\begin{array}{c}29 \\
(3,5)\end{array}$ \\
\hline Total & 499 & 324 & 823 \\
\hline
\end{tabular}


Para que uma terapêutica tenha sucesso, é preciso que haja cooperação do paciente, seja no seguimento às orientações, seja na assiduidade às consultas médicas. No ano de 1992, os hansenianos seguidos no Município de Ribeirão Preto mostraram-se assíduos, com 83,8\% dos pacientes com comparecimento regular aos serviços de atendimento, ligeiramente maior entre os pacientes do sexo feminino $(84,6 \%)$ que entre o sexo masculino $(83,3 \%$ ) (Tabela V). Entre aqueles em registro ativo ao final de 1992, a proporção de doentes com comparecimento regular atingiu $89,12 \%$ (estão excluídos aqueles pacientes que eram irregulares e tiveram saída do registro durante o ano).

Tabela V - Distribuição dos hansenianos atendidos no Município de Ribeirão Preto, segundo sexo e comparecimento às unidades de atendimento no ano de 1992.

\begin{tabular}{lccc} 
Comparecimento & masculino & feminino & Total \\
\hline Regular & 416 & 274 & 690 \\
& $(83,3)$ & $(84,6)$ & $(83,8)$ \\
Não regular & 20 & 14 & 34 \\
& $(4,0)$ & $(4,3)$ & $(4,1)$ \\
Sem atendimento & 37 & 18 & 55 \\
& $(7,4)$ & $(5,5)$ & $(6,7)$ \\
Sem informação & 26 & 18 & 44 \\
& $(5,2)$ & $(5,5)$ & $(5,3)$ \\
Total & 499 & 324 & 823 \\
\hline
\end{tabular}

Os serviços falharam na prevenção do surgimento de incapacidades, fato evidenciado quando se verifica que apenas $46,28 \%$ dos pacientes em registro ativo, até o final de 1992, foram avaliados com relação às incapacidades neurológicas instaladas em mãos, pés e olhos. Entre os casos novos, cerca de $67,36 \%$ dos pacientes foram avaliados, índice considerado baixo pelo Ministério da Saúde, denotando baixa qualidade dos serviços prestados. Sem avaliações periódicas não se pode realizar, eficientemente, a prevenção de seqüelas.

No gráfico 1, estão esquematizadas as freqüências de incapacidades em mãos, pés e olhos nos hansenianos atendidos em Ribeirão Preto em 1992, segundo o sexo e grau de incapacidade. Entre os pacientes avaliados, a presença de incapacidades foi maior no sexo masculino em relação a todas as seqüelas. Deformidades em mãos foram notadas em $6,4 \%$ dos homens e em $2,5 \%$ das mulheres, e deformidades em pés, em 5,8\% dos homens e 5,8\% das mulheres. Havia cegueira em $2,8 \%$ dos homens e $1,8 \%$ das mulheres. Tais valores são altos, e a ineficácia da assistência oferecida fica evidente quando se tem que a proporção de doentes com deformidades, entre os casos com registro ativo avaliados, atinge 47,8\%. Mesmo os pacientes que saem do registro por cura não são satisfatoriamente avaliados, e assim não se conhece a eficácia da assistência oferecida. Apenas 57,69\% dos pacientes com alta por cura foram avaliados, e destes, $60,0 \%$ portavam incapacidades de graus II e III .

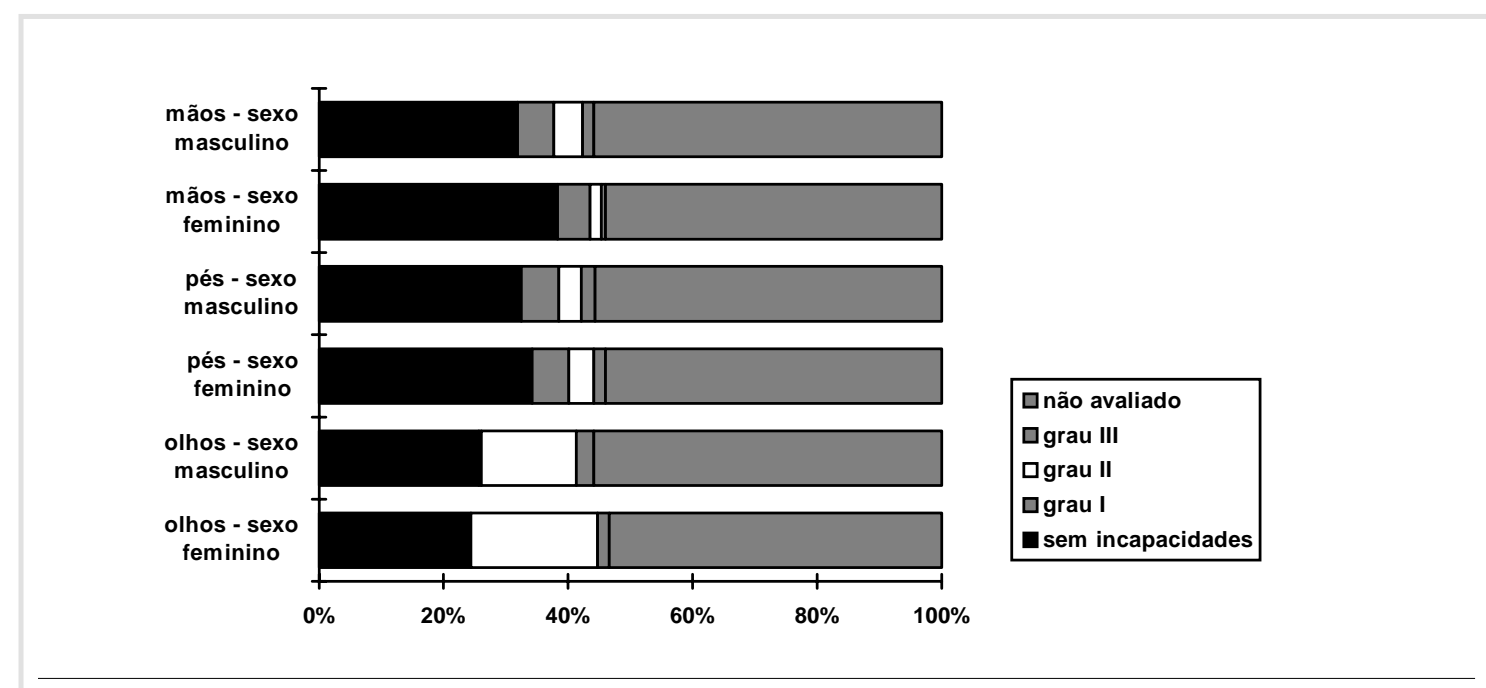

Gráfico 1 - Incapacidades em hansenianos no Sub-Programa de Controle da Hanseníase, Ribeirão Preto-S.P., 1992. 
No ano do estudo, 69 pacientes saíram de registro; $26(3,2 \%)$ pacientes tiveram alta por cura; 20 pacientes foram a óbito, 13 pacientes foram transferidos do Município, 9 tiveram saída administrativa ou alta estatística, e em um caso constatou-se erro diagnóstico.

Percebe-se assim que, frente a uma endemia de altos índices, a assistência é precária; diagnósticos são feitos tardiamente, há falta de cuidados para a prevenção do surgimento de incapacidades e o paciente, dificilmente, tem cura sem seqüelas, apesar de o tratamento ser eficiente na destruição do bacilo. Para o doente, o surgimento de seqüelas incapacitantes é compreendido como perda irreparável, esteja ele com baciloscopia positiva ou negativa. Há cooperação satisfatória da maioria dos pacientes, mas não se encontrou, em 1992, estrutura capaz de fornecer assistência adequada ao hanseniano.

\section{CONCLUSÕES}

Passo a passo com a implantação da PQT, é necessário reformular a assistência ao hanseniano. Reavaliações clínico-neurológicas dos pacientes devem ser feitas uma a duas vezes por ano, com rigorosa vigilância ao surgimento de incapacidades, e com adequadas medidas de prevenção. A exemplo das sessões em grupo que se propõe para a tomada de doses mensais de PQT, é interessante usar o recurso dos gru- pos terapêuticos para organizar a assistência. A abordagem do paciente, após os contatos individuais, em uma situação de grupo inicia um processo de tomada de consciência de sua situação como hanseniano, e permite melhor discussão e abordagem de soluções para seus problemas. Orientações sobre a doença, e sobre saúde, em geral, podem ser fornecidas, transformando o doente em um agente de saúde em sua comunidade. Psicoterapias de ventilação podem ser realizadas. A fisioterapia tem melhor aceitação.

Outra consideração importante para melhor organização da assistência é aquela que se delineia com a implantação do Sistema Único de Saúde (SUS). Haveria recursos humanos e financeiros suficientes, ao passo que a assistência deveria ser centralizada nas Unidades Básicas de Saúde (UBS), por estarem mais próximas da comunidade, facilitando a adesão ao tratamento e atividades de vigilância epidemiológica e de busca ativa. Diagnósticos mais apurados e complicações da terapêutica devem ser encaminhados a serviços com maiores recursos (níveis secundário e terciário), mas a terapêutica pode e deve ser conduzida na própria UBS.

Apenas com melhor estrutura de assistência pode-se tentar erradicar a hanseníase no Brasil; a endemia atual é fruto de uma população desassistida em suas necessidades há séculos, dentro e fora dos serviços de saúde. A hanseníase é um problema de saúde que não pode ser resolvido, separadamente, do contexto sócio-econômico e cultural.

OLIVEIRA SN et al. Epidemiological evaluation of leprosy and leprosy health services in Ribeirão preto City, Brazil, in 1992. Medicina, Ribeirão Preto, 29: 114-122, jan/mar 1996.

ABSTRACT: Analysis of governmental data collected in health services of Ribeirão Preto City in 1992 was made with the purpose of evaluate the leprosy epidemiology and the efficacy of leprosy health services.

It was found a prevalence of $1,72 / 1000$ habitants, that was lower than the brazilian national prevalence in $1992(1,78 / 1000$ habitants), and higher than the W.H.O. definition for low prevalence areas $(0,2 / 1000$ habitants). The majority of patients $(65,4 \%)$ was multibacillary leprosy, and the predominant therapeutics was monoquimiotherapy (despite the W.H.O. recomendations); there was satisfactory patient attendance, but approximately the half of the patients had not evaluations of their disabilities in the year, and $60 \%$ of them had sequelae.

The proposition is to centralize the assistance in Basic Health Care Facilities, approximating the service to community; the participation of medical specialists and more advanced technology would be employed only to improve the diagnosis or in complications in the course of the treatment. The therapy would be applied in groups of patients, in search for social reintegration and health education.

UNITERMS: Leprosy. Public Health. National Health Programs - Evaluation 


\section{REFERÊNCIA BIBLIOGRÁFICA}

1 - BRASIL. Secretaria do Estado de São Paulo. Divisão Técnica de Vigilância Epidemiológica de DST/AIDS/Hanseníase. CVE. Relatório da situação da endemia hansênica no Estado de São Paulo, São Paulo, 1993.

2 - BECHELLI LM \& CURBAN GV. Infecções bacilares da pele. In: Compêndio de dermatologia. 4. ed. Atheneu, São Paulo, p. 120-125, 1975.

3 - CRISTOFOLINI L Prevenção de incapacidades na hanseníase. Reabilitação em hanseníase - Centro de Estudos "Dr. Reynaldo Quagliato". Hospital Lauro de Souza Lima 4. ed. Bauru/SP, p. 1-10, 1982.

4 - BRASIL. Ministério da Saúde. Manual do Subprograma de Controle da Hanseníase, Brasília, 1993.

5 - BRASIL. Fundação Nacional de Saúde / Centro Nacional de Epidemiologia. Plano de eliminação da hanseníase no Brasil para o período de 1995-2000. Informe Epidemiológico do SUS, Ano III, n. 2, FNS, CENEPI, Brasília, maio de 1995.
6 - SEMEGHINI UC A região administrativa de Ribeirão Preto. In: São Paulo no limiar do século XXI. Cenários da Urbanização Paulista. Regiões Administrativas. Governo de São Paulo, Secretaria de Planejamento e Gestão, Fundação SEADE, São Paulo, p. 157-195, 1995.

7 - BRASIL. Instituto Brasileiro de Geografia e Estatísticas. Censo Demográfico 1991. FIBGE, Rio de Janeiro, 764 p, 1991.

8 - RIBEIRÃO PRETO. Plano Diretor da Saúde, Município de Ribeirão Preto, S.P., 1994.

9 - DENISTON OL et al. Evaluación del rendimiento de programas de salud. Bol Of Sanit Panam 67: 389-399, 1969.

10 - SCHOUT D. Municipalização da vigilância epidemiológica: uma proposta em construção. Saúde Soc 3 : 11-28, 1994.

11 - De Rojas V; Hernandez O \& Gil R. Algunos factores que inciden en la demora en el diagnóstico de la lepra. Bol Of Sanit Panam 116: 307-312, 1994.

Recebido para publicação em 27/07/95

Aprovado para publicação em 26/04/96 